\title{
IMPROVING SIMULATION RESULTS WITH STATIC MODELS
}

\author{
Martin J. Miller \\ Capability Modeling, LLC \\ 3113 Coventry E \\ Safety Harbor, FL 34695, USA
}

\author{
Niloo Shahi \\ Olive View-UCLA Medical Center \\ 13442 Olive View Dr. \#2C155 \\ Sylmar, CA 91342, USA
}

\author{
Ashley N. Dias \\ HKS, Inc. \\ 1919 McKinney Avenue \\ Dallas, TX 75201, U.S.A.
}

\begin{abstract}
Effective simulation models require robust development methodologies. Planning, design, data, and testing are integral to ensure valuable answers to the model's customers. This paper discusses how supporting static models provide guidelines and directional correctness to simulation models. Static models can also provide supplemental answers which allow the reduction in simulation model complexity.
\end{abstract}

\section{SIMULATION DEVELOPMENT}

\subsection{Planning and Design}

Simulation is one of the most widely used analytical techniques by professionals in Operations Research and Management Science (Law and Kelton 1991). Simulation analyzes the behavior of either real or imaginary systems over time and is usually performed on a computer using either off-the-shelf or customized software (Crosslin 1995). However, building valid, large scale simulation models usually require months of project effort. The phases of a simulation project typically are as follows.

- Develop a conceptual model

- Program the simulation and user interface software

- Test the software

- Experiment with alternative scenarios

- Present the results to project stakeholders.

\subsection{Data Analysis}

Effective models also require sufficient, valid data which project team members must collect (Miller et al. 2007). This data may come from information systems databases, observations, paper-based charts, and estimates from subject matter experts. Projects may require additional time for data collection due to system complexity (Miller et al. 2006). Also, analysts may need to reformat data for increased usability. These efforts ensure model validity when comparing model results with current processes. 


\subsection{Code and Test}

Projects may follow a phased approach to building simulation models (Miller, Ferrin, and Shahi 2009). The developers fully unit test each phase of code before beginning the next phase of coding. The first phase of the model generates entities in the right quantity and arrival pattern. The second phase involves creating various locations and routing patients using attributes or probabilities, such as acuity levels. In the third phase, developers add resources and activities, which seize and release these resources for specified durations. The next phase includes coding key performance indicators (KPIs) such that the model collects well defined results for analysis. Additional phases may include coding a compelling animation and a graphical user interface (GUI). After these phases are complete, the developer now system tests the components together and fixes all logic and performance issues. The developer ensures model results match expected results. For example, a current state model of an Emergency Department should have Length of Stay (LOS) very close to the LOS of the actual system.

\section{STATIC MODELS}

\subsection{Spreadsheets}

Spreadsheets are one of the most common and flexible computer applications in today's business world. Spreadsheet strengths exist in their ease of use and universal availability. Analysts can build queuing models with spreadsheets and quickly change parameters to test alternative scenarios (Grossman 1999). Although they offer tremendous power and capability, they cannot solve complex dynamic models by themselves. Spreadsheets cannot account for changes in a complex system over time and they neglect variability in such forms as arrival rates, processing times, travel times, resource schedules/failures, etc. (Grabau 2001). Example uses of spreadsheets for simulation projects may include:

- Manipulate and organize complex data sets

- Analyze data sets (i.e., descriptive statistics, graphical analysis)

- Export capabilities (both for the Linear Program \& Simulation models)

- Set up "What If?" scenarios

- Import capabilities (repository for outputs / scenarios)

- Analyze simulation results

- Report capabilities

- Utilize macro capabilities

\subsection{Process Maps}

A process can be defined as a sequence of activities or tasks which achieve a result. Therefore, a process map is simply a structured and documented representation of that process. Examples include drawings, flowcharts, etc. Process maps provide design guidance to a simulation model similar to how a blueprint provides design guidance to building construction (Miller, Pulgar-Vidal, and Ferrin 2002). For example, hospital process maps usually involve all patients routing and activities from arrival to departure. Software applications for process mapping are readily available, extremely easy to use and do not require any understanding of mathematics or programming. Process maps, like most spreadsheets, are said to be "static" representations because they do not account for real-world uncertainty (i.e., probability distributions) or the cause-effect behavior of processes over time. For example, a user typically cannot predict end-to-end process cycle times by looking at a process map.

\subsection{Supplementing the Simulation Model}

The complexity of the problem and detail of the solution usually determines whether spreadsheets or simulations provide the best tool for users (Seila 2003). Small, prototype models used to understand general system behavior can be built with spreadsheets. 


\section{Miller, Shahi, and Dias}

Recall our phased approach to model development (see section 1.1). The first two phases (entities, locations and routings) involve only static parameters. A static model provides guidelines for annual patient volumes at each location. For example, assuming we reserved trauma beds for trauma patients and an emergency department receives 50,000 patients per year and $2 \%$ are trauma patients, then the trauma beds should receive 1,000 patients per year. The developer can similarly follow this logic through for Main Emergency Department (ED) patients, Observations patients, Fast Track Patients, etc. Therefore, a developer can build a static model for given total volumes, the locations that these entities flow to, and the business rules for routing patients. Static model results provide quantifiable targets while unit testing the simulation model.

Determining resource needs and expected utilization using a static model inherently introduces more error with its solution because process variability causes unforeseen queuing (Miller, Ferrin, and Szymanski 2003). However, these results which are based on averages can be adjusted with peak level factors, which offset some of this error. Recall our third phase of simulation model development, where the software developer adds resources and activities that seize and release these resources for specified durations. Resources that are seized by entities for longer durations have higher utilization, by definition. The static model calculates average census at a location by multiplying the volume of entities by their average time at that location. For example, if an Emergency Department keeps 10 patients per day for observations and their average stay in an Observations bed is 12 hours, then the average census for Observations beds is 5. The department may decide to only reserve 5 Observations beds. However, if most observations patient arrive everyday between $8 \mathrm{AM}$ and $10 \mathrm{AM}$, then the census becomes higher and the department will notice frequent queuing for these 5 beds. Increasing the patient volume from an average of 10 to a peak of, say 20, raises the average census to 10 . The department can then choose to reserve between 5 and 10 beds. Determining the proper number requires calibration through trial and error, using sources such as historical data, simulation models and good old fashioned experience.

PROJECT EXAMPLE: EMERGENCY DEPARTMENT

\subsection{Hospital Background}

Olive View-UCLA Medical Center is one of three major public facilities under Los Angeles County Department of Health Services, which is the second largest public health system in the nation. The system is governed by Los Angeles County Board of Supervisors. The facility originally opened in the 1920's as a tuberculosis sanitarium. By the 1940's it was the largest TB sanitarium in the western United States. However, once drugs were developed to treat TB, the population rapidly declined and Olive View became a general hospital in 1959, with a focus on cardiac disease and indigent patients with mental illness.

Olive View also began its partnership with UCLA School of Medicine in 1987 to operate an academic medical center. Presently, there are 29 residency training programs in operation at the hospital, with over 200 residents in training, 600 Physician Attending Staff, 350 UCLA Faculty, 537 Medical Student Rotations and 41 Nursing Student Rotations. The training programs are all run in concert with the David Geffen School of Medicine at UCLA. The facility is a recipient of Award of Excellence in Medical Education from UCLA School of Medicine with Over 1400 applicants for 28 positions. Olive View-UCLA has the only "Center Of Excellence" in the nation for Chagas which is a deadly parasitic disease that is prevalent but unrecognized in USA. The hospital is presently licensed for 377 beds. Of these, 297 are general acute care and 80 are acute Psychiatric beds. Table 1 shows KPIs for the hospital.

Olive View will open an additional 15 general acute care beds in the spring of 2011 to house longterm tuberculosis and other infectious disease patients. The hospital also operates a basic Emergency Room, which moved into a new 51 bed State of the art Emergency Room in March 2011. 


\subsection{Process Challenges}

The old Emergency Room was designed for only 15 beds. However, for more than 35 years of drastic increases in ED volume, it regularly accommodated more than 40 Non-Trauma patients daily in the same limited capacity. In 2010, the emergency room received 84,000 patients. Of these, $8 \%$ Left Before Seen (LBS) due to variety of reasons. The facility made several process changes over time to increase capacity while anxiously awaiting the completion of construction of the new Emergency Room. Some of these efforts included extending capacity by creating an 8 exam room Medical Walk In clinic whereby patients with lower acuity were referred after triage and assessment. This area generated about 23,000 visits a year in addition to Emergency Room (ER) visits. Eventually, this area also reached full capacity, creating unacceptable levels in ED KPIs such as ED LOS and LBS rates.

Table 1: Facility Key Performance Indicators

\begin{tabular}{|l|l|}
\hline \multicolumn{1}{|c|}{ KPI } & \multicolumn{1}{c|}{ Previous Levels } \\
\hline Average Daily Census & 191 patients \\
\hline Average Length of Stay & 4.6 hours \\
\hline Total Admissions & 14,285 patients \\
\hline ER Visits & 45,422 patients \\
\hline Psych ER Visits & 5,666 patients \\
\hline Outpatient Visits & 215,187 patients \\
\hline Medi-Cal Denied Days & $5.9 \%$ \\
\hline All Cause 30 Day Readmission & $5.25 \%$ \\
\hline Mortality Index & 0.82 \\
\hline
\end{tabular}

Numerous initiatives were undertaken to address the escalating patient flow issues, such as:

- Decrease Triage to Medical Screening Exam (MSE) time

- Reduce LOS of acuity 3 patients (on a scale of 1-5)

- Expedite MSE initiation for non-cardiac acuity 3 patients by Medical Walk In physicians

- Initiate "super track" and "treat and street" for all acuity 3 patients

Combinations of these efforts made some improvements in the KPIs (see figure 1). However, the numbers did not reach acceptable targets nor did they sustain. The critical issue remained as "lack of space" in the old area. The frustration with lack of space coupled with the upward trend in volume specifically in the lower acuity patients developed a sense of competition between Medical Walk-In/ Urgent Care (MWI/UC) and ER staff for space in the new facility.

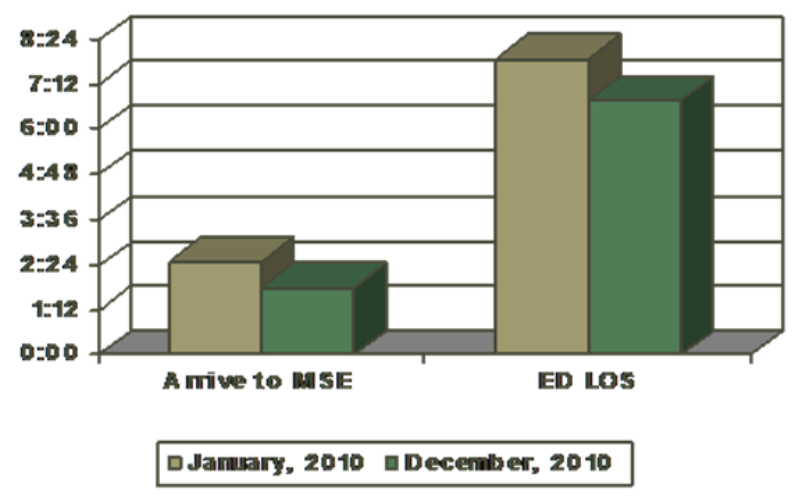

Figure 1: Impact of combining process improvements 


\subsection{Static Model of the ED}

Months before the expected move, the decision for appropriate space allocation of a 51 bed facility became even more challenging. Everyone in the organization realized the great need for an observation area to house short stay patients in order to improve turnaround time of ER beds for incoming new patients. Again, location and space was a pressing issue.

Due to a delayed project start, the Simulation Team could not complete the simulation model prior to opening of the new Emergency Room. Nonetheless, hospital administration needed immediate answers, even if those answers were only directionally correct. The team created a static model determining the necessary capacity for each resource pool of ED beds (Trauma, Main ED, Observations, and Urgent Care). This was done by moving the arrival volume logically through the sequence of patient process, using acuity as the primary decision variable. The average daily volume was then contrasted with a peak volume, using the daily arrival pattern by hour of day. Finally, the census levels for both average and peak times were weighted to provide a best estimate of the beds needed.

Based on these eye opening results, administration made a decision to allocate 8 to 10 beds to Urgent Care while ER receives access to the remainder of the 51 beds, while assigning an area in the old facility for observation patients. In addition to determining the right flow in the new location a sense of urgency for accurate space assignment became eminent for planning of the move.

\subsection{Comparisons to Simulation Model}

The static model predictions, adjusted with peak factors, proved directionally correct. The Project Team completed the simulation model three weeks after the new emergency department opened. The model was calibrated to the new system, which allowed experiments with many scenarios without impacting the existing quality of patient care (Miller, Ferrin, and Szymanski 2003).

Upon completion of the project, the team compared results between the two types of models. The static model underestimated ER census when using average daily volumes and average patient LOS. However, the static model overestimated the census when using either peak volumes or peak LOS. The most accurate proportion was found somewhere between the average and the peak. Ultimately, though, the simulation results reinforced hospital administration's decisions for space allocation, proving the need for keeping the existing 8 beds active for Urgent Care.

\section{PROJECT EXAMPLE: INPATIENT TOWER}

\subsection{Hospital Background}

Orlando Regional Medical Center (ORMC), an 808-bed hospital in downtown Orlando and is Central Florida's only Level One Trauma Center. ORMC specializes in trauma care, critical care, emergency care, cardiology, orthopedics and neurosciences. Additionally, ORMC provides diagnostic and laboratory testing, medical and surgical services, intensive and progressive care, and wound management.

In preparation for a $\$ 250$ million expansion, ORMC conducted a twofold pre-design exercise with the intent to improve the operational efficiency of the expansion project. HKS Architects focused on space programming and lean operations planning through static spreadsheet models, while the project team developed dynamic Lean Six Sigma simulation models that validated and improved the static planning results.

\subsection{Process Challenges}

The hospital recognized the importance of right-sizing their emergency department, inpatient units, and diagnostic and treatment areas due to related impact on boarding time, capacity and flow. ORMC wanted a cutting edge building addition that integrated operations with design in order to demonstrate improved patient and staff satisfaction and yield return on capital investment. 
ORMC undertook this sophisticated planning effort in the midst of potential significant change in the U.S. healthcare delivery system. The recently passed Health Reform is in response to increasing healthcare costs and consumer demand for value - higher quality at a lower cost. The intent is to implement payment reforms to incentivize hospitals and physicians to work together ["bundled payments"] to lower the cost of care. The implications for providers are to expect a reduction in inpatient hospitalizations and an increase in outpatient care, to phase in over 5-10 years. Facility and operations planning in this environment is about choices for capital allocation. The final facility and operations plan not only needed to include a full set of future facility requirements, but also needed to consider options, phasing, priorities, operating efficiency and capital cost, and return on investment. The ability to weigh these variables and to lead ORMC through an informed decision making process was critical to the successful outcome of the planning process.

\subsection{Static Model of the IP Tower}

HKS Architects collaborated with ORMC to review the existing department conditions. They used fiscal year 2010 patient data to predict room and bed needs based on the hospital's preferred and anticipated annual growth rates by service line. They also developed space programs for areas of the hospital that were determined to be part of the expansion project. One challenge of the project, however, was the accelerated timeline that required space programming to begin before operational and simulation results could become available. The team used spreadsheet planning models as a catalyst for the space programming effort. The spreadsheet models used average inpatient LOS and daily volumes as a basis for computing the average daily census. These computations can usually be done quicker than simulation models, however they are not as accurate because they don't account for variability in patient arrivals. Therefore, knowing that averages tend to underestimate capacity, the team compensated by adding an adjustment factor, based on experience.

\subsection{Comparisons to Simulation Model}

The room and bed need established by the static models was validated and revised during space programming by simulation modeling. In addition to identifying process and capacity improvement opportunities, simulation of projected 2020 volumes provided guidance on a series of spatial and operational concerns for each area. The inpatient model tested preferred utilization targets for acute, intermediate and critical care units and percentage and number of beds in each acuity and service line.

The model also identified resource bottlenecks, evaluated scenarios and best practices based on rising volumes to determine resources needed to remove bottlenecks, and showed critical occupancy levels to support capacity design. Eleven improvement opportunities were identified from the simulation modeling for ORMC as worth implementing due to their potential impact. The inpatient opportunities included improving Discharge Time of Day, create Inpatient Discharge Lounge and create Inpatient Admissions Unit.

The project team found the static model overestimated the number of inpatient beds by an average of 15 percent, with a range of 0 to 45 percent by unit. The largest positive impact though, was made when the inpatient units were combined within the new facility to create fewer, less specialized units. The removal of specialization allowed for larger groups of beds and fewer transfers, which in turn decreased wait time for a bed and decreased the overall inpatient LOS. This added flexibility in the process had a dramatic and positive impact on the flow of patients. Using simulation results, hospital management can begin mitigating risks and solving issues months before they transition to the new facility.

\section{CONCLUSION}

Hospitals build and expand their facilities intending to improve their patient throughput. Hospital executives need to know they've designed their new facilities with sufficient capacity. They also like to know how each process improvement increases capacity and throughput. Simulation provides the best tool for solving these challenging problems (Miller, Ferrin, and Shahi 2009). If project timeframes don't allow 
for development of a proper simulation model, then static models are a useful, common sense alternative. Even if sufficient timeframes exist, creation of static models can support simulations. Static models can provide directional correctness for simulation answers and new insights into the system's behavior. Finally, project management should always set client expectations regarding the expected quality of their answers with the goal of providing the best possible deliverables in the timeframes available.

\section{REFERENCES}

Crosslin, R. 1995. "Simulation, The Key to Designing and Justifying Business Reengineering Projects." In The Electronic College of Process Innovation. U.S. Department of Defense.

Grabau, M. R. 2001. "Averages Kill (Or How to Sell Business Process Simulation)." In Proceedings of the 2001 Winter Simulation Conference, edited by B. A. Peters, J. S. Smith, D. J. Medeiros, and M. W. Rohrer, 1262-1265. Piscataway, New Jersey: Institute of Electrical and Electronics Engineers, Inc.

Grossman, T. A. 1999. "Spreadsheet Modeling and Simulation Improves Understanding of Queues." Interfaces 29(3): 88-103. Templates available from http://www.ucalgary.ca/ grossman/simulation/.

Law, A. M., and W. D. Kelton. 1991. Simulation Modeling and Analysis. New York, NY: McGraw-Hill.

Miller, M., F. Pulgar-Vidal, and D. Ferrin. 2002. "Achieving Higher Levels of CMMI Maturity Using Simulation." In Proceedings of the 2002 Winter Simulation Conference, edited by E. Yücesan, C.-H. Chen, J. L. Snowdon, and J. M. Charnes, 1473-1478. Piscataway, New Jersey: Institute of Electrical and Electronics Engineers, Inc.

Miller, M., D. Ferrin, and J. Szymanski. 2003. "Simulating Six Sigma Improvement Ideas For A Hospital Emergency Department." In Proceedings of the 2003 Winter Simulation Conference, edited by S. Chick, P. Sanchez, D. Ferrin, and D. Morrice, 1926-1929. Piscataway, New Jersey: Institute of Electrical and Electronics Engineers, Inc.

Miller, M., D. Ferrin, T. Flynn, M. Ashby, K. White, and M. Mauer. 2006. "Using RFID Technologies To Capture Simulation Data In A Hospital Emergency Department." In Proceedings of the 2006 Winter Simulation Conference, edited by L. F. Perrone, F. P. Wieland, J. Liu, B. G. Lawson, D. M. Nicol, and R. M. Fujimoto, 1365-1371. Piscataway, New Jersey: Institute of Electrical and Electronics Engineers, Inc.

Miller, M., D. Ferrin, M. Ashby, T. Flynn, and N. Shahi. 2007. "Merging Six Emergency Departments Into One: A Simulation Approach." In Proceedings of the 2007 Winter Simulation Conference, edited by S. G. Henderson, B. Biller, M.-H. Hsieh, J. Shortle, J. D. Tew, and R. R. Barton, 1574-1578. Piscataway, New Jersey: Institute of Electrical and Electronics Engineers, Inc.

Miller, M., D. Ferrin, and N. Shahi. 2009. "Estimating Patient Surge Impact on Boarding Time in Several Regional Emergency Departments." In Proceedings of the 2009 Winter Simulation Conference, edited by M. D. Rossetti, R. R. Hill, B. Johansson, A. Dunkin, and R. G. Ingalls, 1906-1915. Piscataway, New Jersey: Institute of Electrical and Electronics Engineers, Inc.

Seila, A. F. 2003. "Spreadsheet Simulation." In Proceedings of the 2003 Winter Simulation Conference, edited by S. Chick, P. J. Sánchez, D. Ferrin, and D. J. Morrice, 25-30. Piscataway, New Jersey: Institute of Electrical and Electronics Engineers, Inc.

\section{AUTHOR BIOGRAPHIES}

MARTIN J. MILLER is currently a Partner with Capability Modeling, LLC. He previously worked over ten years as co-founder and Director at Business Prototyping, FDI Healthcare and Northern Lights. He worked exclusively in the healthcare industry improving patient throughput and developing simulation models and analysis. He also worked over eight years for Accenture and was a Manager for their Capability Modeling And Simulation practice. He obtained his CMM Certification from the Software Engineering Institute in 1998. He received his Masters of Science in Industrial \& System Engineering and 
Bachelors of Science in Aerospace Engineering from the University of Florida. His email address is mmiller@capabilitymodeling.com.

NILOO SHAHI is Associate Hospital Administrator II, holding the Chief of Staff and Operations title at Olive View-UCLA Medical Center. She has a Doctorate Degree in Public Health Administration from UCLA. In addition, she holds Six Sigma Master Black Belt Certification from American Society for Quality. She has over 20 years of experience in facility operations and process improvement with various healthcare institutions mainly in the Los Angeles County- Public Hospitals. Her email address is nshahi@dhs.lacounty.org.

ASHLEY N. DIAS is an operational healthcare planner with HKS Architects. She earned her Master of Architecture with a Certificate in Health Systems and Design from Texas A\&M University in 2008. She has participated in conceptual design, schematic design, design development, and construction documentation, as well as research-related activities with Clinical Solutions \& Research group of HKS Architects. Her email address is adias@hksinc.com. 